\title{
ARTICLE OPEN \\ Topological networks for quantum communication between distant qubits
}

\author{
Nicolai Lang ${ }^{1}$ and Hans Peter Büchler ${ }^{1}$
}

Efficient communication between qubits relies on robust networks, which allow for fast and coherent transfer of quantum information. It seems natural to harvest the remarkable properties of systems characterized by topological invariants to perform this task. Here, we show that a linear network of coupled bosonic degrees of freedom, characterized by topological bands, can be employed for the efficient exchange of quantum information over large distances. Important features of our setup are that it is robust against quenched disorder, all relevant operations can be performed by global variations of parameters, and the time required for communication between distant qubits approaches linear scaling with their distance. We demonstrate that our concept can be extended to an ensemble of qubits embedded in a two-dimensional network to allow for communication between all of them.

npj Quantum Information (2017)3:47; doi:10.1038/s41534-017-0047-x

\section{INTRODUCTION}

Systems characterized by topological invariants are well known to exhibit unique properties with potential applications in quantum information processing and engineering. ${ }^{1}$ Ever since the first experimental observation of the integer quantum Hall effect, ${ }^{2-4}$ many other condensed matter systems have been identified and experimentally characterized, such as fractional quantum Hall fluids $^{5-7}$ and topological insulators and superconductors. ${ }^{8-16}$ The latter belong to a particularly well-understood family of topological systems described by non-interacting fermions, where topological invariants can be defined on classes of random matrices. ${ }^{17-20}$ This concept can be straightforwardly generalized to bosonic setups, as well as classical systems, ${ }^{21-23}$ where the topological features still give rise to intriguing properties, such as localized and chiral edge modes. Here, we are interested in such systems: We demonstrate that their topological properties can be harvested for robust and efficient transfer of quantum information over large distances.

Several different platforms for the realization of topological systems of artificial matter with bosonic degrees of freedom are currently explored: The construction of topological band structures and the observation of edge states has been achieved with photonic circuits in the optical ${ }^{21,24,25}$ and the radio-frequency ${ }^{26}$ regime, as well as with classical coupled harmonic oscillators, ${ }^{22,23,27}$ and with cold atomic gases. ${ }^{28-34}$ These experimental advances have been prepared and are supported by many theoretical proposals, e.g. refs. 35-45. Several of the above platforms are suitable to carry a single, quantized excitation with low losses and dissipation along protected edge channels, which opens the opportunity to harvest topological phenomena for guiding and transmitting quantum information reliably. First approaches in this direction have been proposed ${ }^{46,47}$ and primarily focus on the transmission of excitations along protected edge modes on the boundary of a two-dimensional, topologically non-trivial medium.
Here, we show that a linear network of coupled bosonic degrees of freedom, characterized by topological bands, can be employed for the highly efficient exchange of quantum information over large distances. We demonstrate the superiority of this setup over its topologically trivial counterparts and exemplify its application with the implementation of a robust quantum phase gate. Our proposal is based on a (quasi) one-dimensional setup, characterized by a $\mathbb{Z}$ topological index, ${ }^{48}$ and derived from paradigmatic systems such as Kitaev's Majorana chain ${ }^{49}$ and the Su-Schrieffer-Heeger (SSH) model. ${ }^{50}$ It features symmetry protected, localized edge modes, the extend and overlap of which can be tuned via coupling parameters to facilitate controllable communication between them. Important features of our setup are that relevant operations can be performed by global variations of parameters, its robustness with respect to the pulse shapes used for the transfer protocol, and that the time for the transfer scales favorably with the separation of the qubits. This high gain in performance is bought by more complex preparation schemes as the coupling parameters have to respect symmetries protecting the topological invariants. ${ }^{21,22,26,27}$ Finally, we demonstrate that our concept can be extended to an ensemble of qubits embedded in a two-dimensional network of local bosonic degrees of freedom to allow for communication between all of them.

We consider macroscopically separated qubits that are coupled by a linear quantum network, see Fig. 1a for an example. The quantum network itself is constructed from bosonic degrees of freedom with only local couplings between them, and generically described by the Hamiltonian

$\hat{H}_{n}=\sum_{i, j} b_{i}^{\dagger} H_{i j} b_{j}$.

Here, $b_{i}^{\dagger}\left(b_{i}\right)$ are bosonic creation (annihilation) operators accounting for the mode at site $i$ with $H_{i j}$ the coupling amplitudes. The network is designed such (see below) that at the end $p$ of each branch, a localized bosonic edge mode $\tilde{b}_{p}$ emerges with a

\footnotetext{
${ }^{1}$ Institute for Theoretical Physics III and Center for Integrated Quantum Science and Technology, University of Stuttgart, 70550 Stuttgart, Germany Correspondence: Nicolai Lang (nicolai@itp3.uni-stuttgart.de)
}

Received: 23 June 2017 Revised: 26 September 2017 Accepted: 29 September 2017

Published online: 07 November 2017 


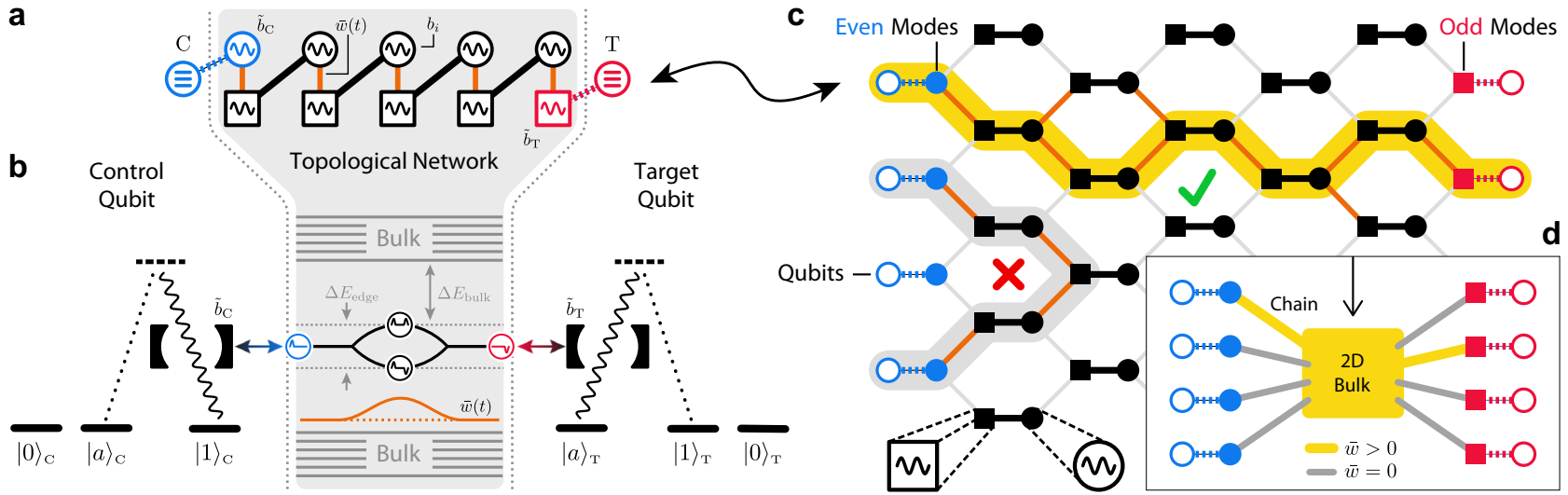

Fig. 1 Topological quantum networks. a One-dimensional chain of bosonic modes $b_{i}$ with globally tunable onsite couplings $\bar{w}(t)$ (orange); derived from the SSH chain. It features a topological band structure with localized edge modes (in the topological phase), which can be coupled to local qubits T and C. b The local qubits are realized as three-level systems with logical states $|0\rangle_{p},|1\rangle_{p}$ and the auxiliary state $|a\rangle_{p}$. The state $|a\rangle_{C}\left(|1\rangle_{T}\right)$ can decay into $|1\rangle_{C}\left(|a\rangle_{T}\right)$ via an off-resonant transition and thereby emits an excitation into the localized edge mode $\tilde{b}_{\mathrm{C}}$ $\left(\tilde{b}_{\mathrm{T}}\right)$. Tunneling excitations between the two edges is facilitated by tuning the chain close to the phase transition via $\bar{w}(t)$ and adiabatically decoupling bulk from edge modes. c Possible 2D generalization of the network on a dimerized honeycomb lattice. Scattering-free transport is guaranteed by topological protection which requires a sublattice symmetry. The latter is realized by directly coupling only "even" (filled circles) with "odd" modes (filled squares). State transfer between qubits (empty circles) of different (the same) type is possible (impossible), illustrated by the bold yellow (gray) path. Stray couplings in the bulk (shown for the upper path) are not detrimental to the transfer fidelity. $\mathbf{d}$ Instead of locating the edge modes (filled colored circles and squares) with their qubits at the boundary, emanating SSH chains can be used to separate the qubits from each other and the 2D bulk. There is no need to trace out a specific path as in c, but a weak addressability of the individual chains is sufficient whereas the couplings of the bulk can be tuned globally. Details are given at the end of the manuscript

controllable coupling between this mode and a local qubit. The conceptually simplest setup to envisage is an optical network coupled to a single atom with the level structure shown in Fig. 1b. There the coupling Hamiltonian for the target qubit $\mathrm{T}$ takes the form (within the rotating wave approximation)

$\hat{H}_{\mathrm{T}}(t)=g_{\mathrm{T}}(t)\left[\tilde{b}_{\mathrm{T}}^{\dagger}|a\rangle\left\langle\left. 1\right|_{\mathrm{T}}+\tilde{b}_{\mathrm{T}} \mid 1\right\rangle\left\langle\left. a\right|_{\mathrm{T}}\right]\right.$.

The coupling $g_{\mathrm{T}}(t)$ is controlled by external laser fields and allows for the application of $\pi$-pulses between the qubit state $|1\rangle_{T}$ and the edge mode $\tilde{b}_{T}$, i.e., the emission of a photon into the edge mode $b_{\mathrm{T}}$ from state $|1\rangle_{\mathrm{T}}$ is accompanied by a transition into the auxiliary state $|a\rangle_{T}$; in the following, we denote such a $\pi$-pulse at edge $p$ by the unitary operation $\Pi_{p}$. Note that the Hamiltonian $\hat{H}_{C}(t)$ for the control qubit $C$ is similar, with the role of $|1\rangle_{C}$ and $|a\rangle_{C}$ exchanged.

Several fundamental quantum information processing tasks between the qubits reduce to the transfer of edge excitations within the linear network; we denote the corresponding unitary operation that describes the transfer of excitations between edges $p$ and $q$ as $T_{p \leftrightarrow q}$. As an example, the protocol for a controlledphase (CP) gate between a control qubit at position $C$ and a target qubit at position $T$ reads

$\mathcal{U}_{\mathrm{CP}}=\Pi_{\mathrm{T}} \circ T_{\mathrm{C} \leftrightarrow \mathrm{T}} \circ \Pi_{\mathrm{C}}^{2} \circ T_{\mathrm{C} \leftrightarrow \mathrm{T}} \circ \Pi_{\mathrm{T}}$.

Another example, the transport of a control qubit to a target position, is simply described by the protocol

$\mathcal{U}_{\text {SWAP }}=\mathcal{A}_{\mathrm{C}} \circ \Pi_{\mathrm{T}} \circ \Pi_{\mathrm{C}} \circ T_{\mathrm{C} \leftrightarrow \mathrm{T}} \circ \Pi_{\mathrm{C}} \circ \Pi_{\mathrm{T}} \circ \mathcal{A}_{\mathrm{C}}$.

Here, $\mathcal{A}_{p}$ denotes the exchange of the two states $|1\rangle_{p}$ and $|a\rangle_{p}$. Note that this operation even performs the full exchange of the two qubits due to the linearity of the network; a detailed discussion of these operations can be found at the end of the manuscript.

Motivated by these observations, we are in the following interested in the efficient transfer $\left(T_{\mathrm{C} \leftrightarrow \mathrm{T}}\right)$ of edge excitations within the linear network. The basic idea is most conveniently illustrated for two qubits coupled by a one-dimensional network as illustrated in Fig. 1a: The structure of the network gives rise to topological bands with a gapped dispersion relation and entails the existence of degenerate and localized edge modes within the bulk gap. As the existence of edge modes is topologically protected, it is robust against disorder. In a finite system, the degeneracy of the topological edge states is only lifted exponentially in the edge separation. However, globally tuning the quantum network closer to the topological phase transition into the trivial phase increases overlap and finite size splitting of the edge states, and eventually allows for a $\pi$-pulse $T_{\mathrm{C} \leftrightarrow \mathrm{T}}$ between the two edges. This simple idea is the core of our protocol; compared to topologically trivial systems, it features several superior properties regarding the speed of state transfer and its robustness against disorder, as well as the absence of individual addressing of each part of the network.

Finally, we would like to stress that our scheme is generic and one can envisage various experimental platforms for its implementation. In addition to the discussed optical network, ${ }^{21}$ alternative setups are coupled optical cavities and circuit quantum electrodynamics systems, ${ }^{51,52}$ as well as trapped polar molecules or Rydberg atoms with a coupling mediated by dipolar exchange interactions, ${ }^{45}$ while the local qubits can be artificial atoms, ${ }^{53}$ nitrogen-vacancy centers in diamond, ${ }^{54}$ or trapped ions. ${ }^{55}$

\section{RESULTS}

Topological network

We start with a description of the requirements on the quantum network $\hat{H}_{n}$ to exhibit topologically protected edge modes in a one-dimensional chain with two edges, as illustrated in Fig. 1a; the generalization to $2 \mathrm{D}$ networks is discussed at the end of the manuscript.

The most prominent paradigmatic model in one-dimension is the Majorana chain, which exemplifies the concept of symmetry protected topological phases, originally formulated for spinless fermions with a mean-field $p$-wave pairing term. ${ }^{49}$ This model is closely related to the Su-Schrieffer-Heeger (SSH) model ${ }^{50}$ in the single-particle picture. It turns out that the necessary steps to translate these models into our bosonic quantum network 

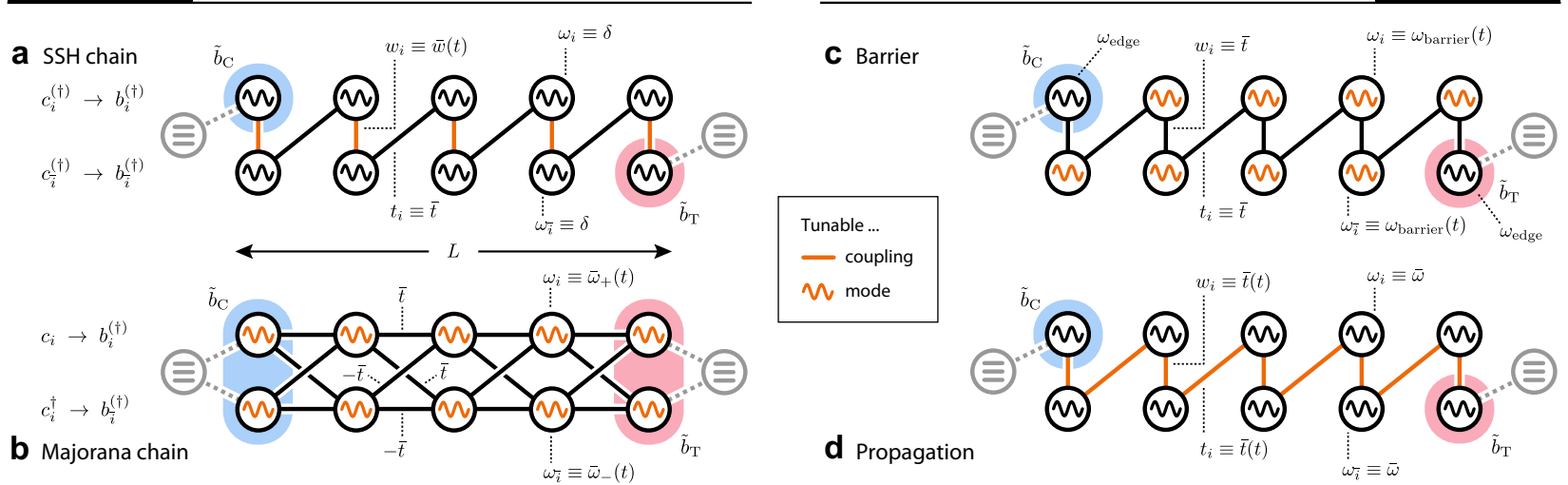

Fig. 2 Setups. Possible setups for state transfer via global control parameters. The left panel depicts networks with topological bands. The models in the right panel are used for comparison and feature only trivial bands. Each model consists of locally coupled bosonic modes with qubits coupled to the edge modes $\tilde{b}_{\mathrm{C}}$ and $\tilde{b}_{\mathrm{T}}$. a The SSH chain inspired setup described by $\hat{H}_{\mathrm{bSSH}}(t)$; globally tunable are only the onsite couplings $\bar{w}(t)$. b Network inspired by the Majorana chain (unitarily equivalent to the SSH setup) and described by $\hat{H}_{\mathrm{bMC}}(t)$; the homogeneous eigenfrequency differences $\delta \bar{\omega}(t) \equiv \bar{\omega}_{-}(t)-\bar{\omega}_{+}(t)$ are tunable. For details we refer the reader to Supplementary Information Section 1. c Model with two artificial edge modes separated by a simple tunneling barrier of tunable eigenfrequencies $\omega_{\text {barrier }}(t)$; described by the Hamiltonian $\hat{H}_{\mathrm{bB}}(t)$. d The simplest model, based on free propagation of excitations and described by $\hat{H}_{\mathrm{bP}}(t)$; all couplings $\bar{t}(t)$ are tuned simultaneously

language are more conveniently performed for the SSH model; the discussion of the Majorana chain and its relation to the SSH chain is presented in the Supplementary Information Sections 1 and 2 .

The SSH model on a chain with $L$ sites and open boundaries is described by the Hamiltonian

$\hat{H}_{\mathrm{SSH}}=\sum_{i=1}^{L} w_{i} c_{i}^{\dagger} c_{\bar{i}}+\sum_{i=1}^{L-1} t_{i} c_{\bar{i}}^{\dagger} c_{i+1}+$ h.c.

with the two fermion operators $c_{i}$ and $c_{\bar{i}}$ on each site. Note that the indices $i$ label the "upper" fermionic modes whereas bar-ed indices $\bar{i}$ denote the "lower" ones, see Fig. 2a; we will use uppercase indices $l$ if we refer to both indifferently. Here, $w_{i}$ and $t_{i}$ are the hopping amplitudes. For a uniform system with $w_{i} \equiv \bar{w}$ and $t_{i}=\bar{t}$, one obtains a gapless point for $\bar{w}=\bar{t}$ separating a topological phase for $\bar{w}<\bar{t}$ from the trivial phase for $\bar{w}>\bar{t}$. The former features topologically protected edge modes, which are fermionic in nature. The second-quantized Hamiltonian can be encoded by a matrix $H_{\mathrm{SSH}}$ via $\hat{H}_{\mathrm{SSH}}=\boldsymbol{\Phi}^{\dagger} H_{\mathrm{SSH}} \boldsymbol{\Phi}$ with the pseudospinor $\boldsymbol{\Phi}=\left(c_{1}, c_{\overline{1}}, \ldots, c_{L}, c_{\bar{L}}\right)^{T}$. For real hopping amplitudes $w_{i}$ and $t_{i}$, the Hamiltonian exhibits time reversal symmetry $T=\mathcal{K}$, where $\mathcal{K}$ denotes complex conjugation. Furthermore, time reversal $T$ together with the sublattice symmetry $S=U_{C}^{S S H}$, represented by the unitary

$U_{C}^{S S H}=1_{L \times L} \otimes\left(\begin{array}{cc}1 & 0 \\ 0 & -1\end{array}\right)$,

yields the particle-hole $(\mathrm{PH})$ symmetry $C=\mathcal{K} U_{C}^{\text {SSH }}$ with $C^{2}=+1$. Hence, the $\mathrm{SSH}$ chain is in symmetry class $\mathrm{BDI}$ of the Altland-Zirnbauer classification. ${ }^{17-20}$ In one dimension, this allows for the definition of a $\mathbb{Z}$ topological invariant, ${ }^{11-14}$ which is responsible for the emergence of the disorder-resilient edge modes bound to the open ends of the chain in the topological phase.

The implementation of an analog system with bosonic degrees of freedom is straightforward: We replace the fermionic operators by bosonic ones, i.e., $c_{l}^{(\dagger)} \rightarrow b_{l}^{(\dagger)}$. The bosonic Hamiltonian takes the form

$\hat{H}_{\mathrm{bSSH}} \equiv \xi^{\dagger}\left[H_{\mathrm{SSH}}+\delta 1\right] \xi \equiv \xi^{\dagger} H_{\mathrm{bSSH}} \xi$ with $\xi=\left(b_{1}, b_{\overline{1}}, \ldots, b_{L}, b_{\bar{L}}\right)^{T}$ and $H_{\mathrm{bSSH}}=H_{\mathrm{SSH}}+\delta 1$. The constant positive energy shift $\delta>0$ is required to enforce positivity on the matrix $H_{\mathrm{bSSH}}$ and accounts for the energy $\omega_{l}$ of each bosonic mode $b_{\text {. }}$. The bosonic Hamiltonian $\hat{H}_{\mathrm{bSSH}}$ features the same single-particle band structure as the original fermionic chain, and exhibits the same topological properties and topological quantum numbers. Therefore, it gives rise to the same edge modes. Note that these are statements about single-particle physics where statistics is not relevant. To satisfy the $\mathrm{PH}$ symmetry, one must respect the sublattice symmetry (6) that protects the topological invariant. This is equivalent to the constraints

$\omega_{i}=\delta=\omega_{\bar{i}}$

for all sites $i$ and $\bar{i}$, i.e., all bosonic modes must have the same energy. Note that there are no constraints on the couplings $w_{i}$ and $t_{i}$.

To complete the picture, we point out that the bosonic realization of the Majorana chain $H_{\mathrm{bMc}}$ is unitarily equivalent to that of the SSH chain $H_{\mathrm{bsSH}}$; this is shown in the Supplementary Information Section 2. However, despite their unitary equivalence, the bosonic networks impose different symmetry constraints on the coupling Hamiltonians. Therefore, depending on the experimental constraints on protecting the symmetry, it may be advantageous to implement one or the other of the unitary equivalent models.

Protocol for state transfer

Next, we discuss the protocol for state transfer. One of the key features of the protocol is that we require only global (translational invariant), time-dependent tuning of the hopping amplitudes $w_{i}$ i in particular, single-site addressability and control is not required. The goal of the protocol is to coherently transfer a single, quantized excitation from one of the localized edge modes to the other by means of an adiabatic variation of the couplings $\bar{w}$ in $\mathrm{H}_{\mathrm{bSSH}}$.

The crucial point we exploit for state transfer is that in finite systems and in the topological phase (for $0<\bar{W}<\bar{t}$ ), there is a finite overlap between the edge modes due to their exponential extension into the bulk. While deep in the topological phase this overlap is exponentially suppressed, it can be strongly enhanced by tuning $\bar{w}$ closer to $\bar{t}$ from below, allowing for tunneling 
between the macroscopically separated edge modes. In order to prevent scattering into bulk modes, edge physics and bulk physics have to be adiabatically decoupled. This can be achieved by tuning $\bar{W}$ smoothly (and slowly, see below) towards the topological transition and return to the "sweet spot" $\bar{w}=0$ afterwards to relocalize (and thereby decouple) the edge modes. To this end, we introduce a time-dependent hopping rate $\bar{w}(t)=$ $\bar{w}_{\max } \mathcal{F}(t)$ giving rise to the time-dependent network Hamiltonian $\hat{H}_{\text {bSSH }}(t)$ with perfectly localized edge modes at $t=0$ and $t=\tau$. For simplicity, we choose for the adiabatic process the smooth pulse shape

$$
\mathcal{F}(t)=\sin ^{2}(\pi t / \tau) \quad \text { for } \quad 0 \leq t \leq \tau .
$$

Here, $\tau$ denotes the characteristic time scale for the pulse. The exact pulse shape does have influence on the performance of the protocol, and setup-specific optimizations may yield quantitatively better results, see below.

We analyze the transfer efficiency and its dependence on the parameters $\tau$ and $\bar{w}_{\max }$ by numerically evaluating the full unitary time evolution. We start with an excitation in the left edge mode $\tilde{b}_{\mathrm{C}}$,

$$
\left|\Psi_{0}\right\rangle=|1\rangle_{1} \otimes|0, \ldots, 0\rangle_{\text {bulk }} \otimes|0\rangle_{\bar{L}} \equiv|1,0,0\rangle,
$$

and are interested in the transfer to the right edge mode $\tilde{b}_{T}$, i.e., the state $|0, \mathbf{0}, 1\rangle$. The transfer is characterized by the overlap

$$
\left\langle 0, \mathbf{0}, 1\left|U_{\tau}\left(\bar{W}_{\max }\right)\right| 1, \mathbf{0}, 0\right\rangle \equiv \sqrt{\mathcal{O}} \mathrm{e}^{i \varphi} \text {. }
$$

Here, $\mathcal{O} \geq 0$ denotes the transfer fidelity, while $\varphi$ is the relative phase accumulated during the adiabatic process. $U_{\tau}\left(\bar{w}_{\max }\right)$ is the unitary time evolution operator at time $t=\tau$, which depends parametrically on $\bar{w}_{\max }$. To quantify the degree of adiabaticity, we introduce another characteristic parameter which describes the total edge mode population,

$\mathcal{E}=\mathcal{O}+\left|\left\langle 1, \mathbf{0}, 0\left|U_{\tau}\left(\bar{w}_{\max }\right)\right| 1, \mathbf{0}, 0\right\rangle\right|^{2}$.

Deviations of $\mathcal{E}$ from unity indicate undesired losses into bulk modes.

The qualitative results for the transfer are shown in Fig. 3a for an optimized set of parameters $\tau$ and $\bar{w}_{\max }$, i.e., slow transfer with $\tau \gg \bar{t}^{-1} \quad(\hbar=1)$. As expected, we find perfect transfer and decoupling of edge and bulk modes. The overall performance is quantified by $\mathcal{O}, \varphi$ and $\mathcal{E}$, and depends on how close the protocol parameter $\bar{w}_{\max }$ is to the critical value $\bar{t}$, the size of the system $L$, and the global time scale $\tau$; see Fig. $4 a, b$ for a chain of length $L=5$. The edge weight $\mathcal{E}$ (gray background tiles) equals unity almost everywhere, except for very fast protocols and tiny bulkedge gaps. We observe quite generally that for a smooth pulse shape like $\mathcal{F}$, the adiabatic bulk-edge decoupling is rather generically established in the topological setup. The size of colored squares denotes the transfer $\mathcal{O}$, while the color accounts for the value of the phase $\varphi$ accumulated during the transfer (measured in the rotating frame of the localized edge modes). We find several disjoint branches with $\mathcal{O} \approx 1$ corresponding to an increasing number of round trips of the excitation; see Fig. $4 \mathrm{~b}$. The outermost branch allows for the fastest and most robust transfer, and is therefore the desired parameter regime to perform quantum operations. However, the most striking property of this setup is a fixed phase $\varphi$ accumulated during a transfer, i.e., $\varphi= \pm \pi /$ 2. The sign depends on the number of round trips and on the parity of the chain length $L$, see Supplementary Information Sections 4 and 5 for an explanation. This remarkable feature is a peculiarity of the $\mathrm{PH}$ symmetric topological setup and in general violated for other setups (see comparison below). A motivation for the relation of $\mathrm{PH}$ symmetry and fixed phase is presented in Supplementary Information Section 4.

As a concluding remark, note that there may be residual couplings $\bar{w}_{\text {min }} \ll \bar{w}_{\text {max }}$ that cannot be switched off for $t<0$ and $t$ $>\tau$. Weak residual couplings $\bar{W}_{\text {min }}$ (compared to $\bar{t}$ ) can be tolerable on the relevant timescales as they are exponentially suppressed with the qubit distance $L$ in the topological setup, while controlled coupling is always possible for $\bar{w}_{\max } \rightarrow \bar{t}$.

\section{Scaling and adiabaticity}

An important aspect for quantum information processing over large distances is the scalability of the protocol with separation $L$ between the qubits. We identify the two relevant time scales of the transfer protocol: The inverse edge mode splitting $\Delta E_{\text {edge' }}^{-1}$ which determines the time for a state transfer between the two edge states, and second, the inverse of the bulk-edge separation $\Delta E_{\text {bulk, }}^{-1}$ which gives a lower bound on the protocol time scale due to the required adiabatic bulk-edge decoupling.

We start by considering the scaling of these energies when the topological phase transition $\bar{w}=\bar{t}$ is approached from the topological phase $\bar{w}<\bar{t}$. In the limit $L \rightarrow \infty$, the eigenvalues of $H_{\text {bsSH }}$ derive from the transcendental equation (see Supplementary Information Section 5 for the derivation)

$$
\frac{\Delta \bar{W}^{\prime}-\sqrt{\Delta \bar{W}^{\prime 2}-\lambda^{\prime 2}}}{\Delta \bar{W}^{\prime}+\sqrt{\Delta \bar{W}^{\prime 2}-\lambda^{\prime 2}}}=\mathrm{e}^{-2 \sqrt{\Delta \bar{W}^{\prime 2}-\lambda^{\prime 2}}}
$$

\section{topological}
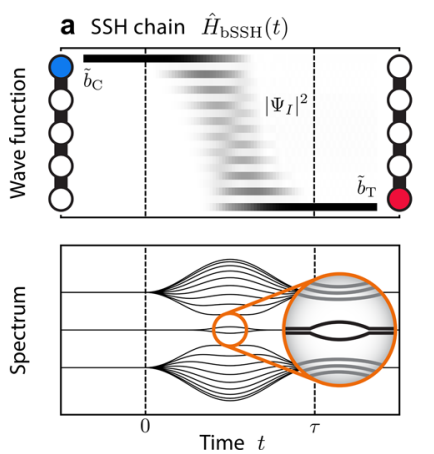

b Majorana chain $\hat{H}_{\mathrm{bMC}}(t)$
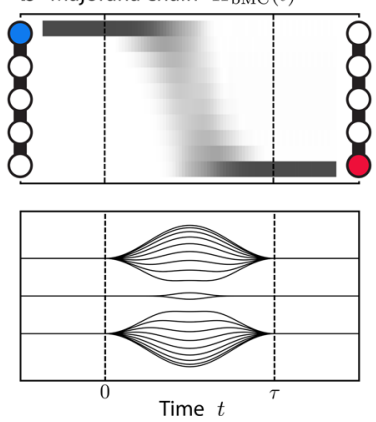

c Barrier $\hat{H}_{\mathrm{bB}}(t)$
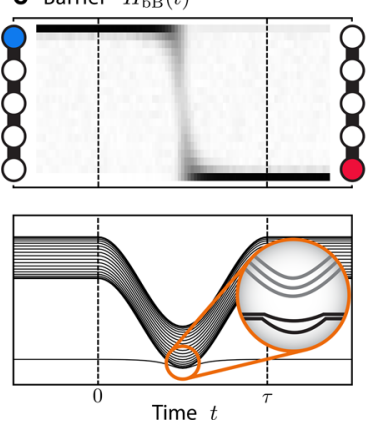

d Propagation $\hat{H}_{\mathrm{bP}}(t)$
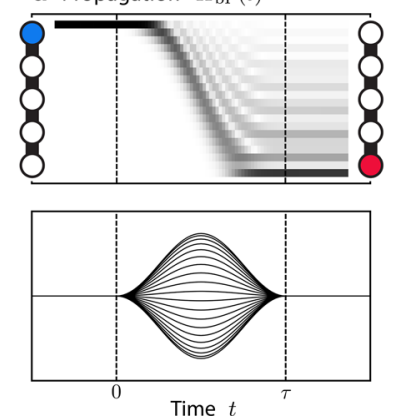

Fig. 3 State transfer-qualitative results. For the four setups depicted in Fig. 2 and the corresponding protocols described in the text, we show the full time evolution of a single excitation that is initially localized in the left mode $\tilde{b}_{\mathrm{C}}$ and transferred to the right mode $\tilde{b}_{\mathrm{T}}$. The protocol parameters $\bar{w}_{\max } \mathbf{a}, \delta \bar{\omega}_{\max } \mathbf{b}, \omega_{\text {barrier }}^{\min } \mathbf{c}, \bar{t}_{\max } \mathbf{d}$ and the time scale $\tau$ are tuned to optimize transfer $\mathcal{O}$ and edge weight $\mathcal{E}$. The upper row shows the amplitude of the single-particle wave function under the time evolution prescribed by $\hat{H}_{\bullet}(t)$. The lower row parallels the time evolution by the single-particle spectrum of $\hat{H}_{\bullet}(t)$, i.e., the spectrum of $H_{\bullet}(t)$. In this work, we focus on the topological setup derived from the SSH chain a and compare it with the trivial setup of a simple tunneling barrier $\mathbf{c}$ 

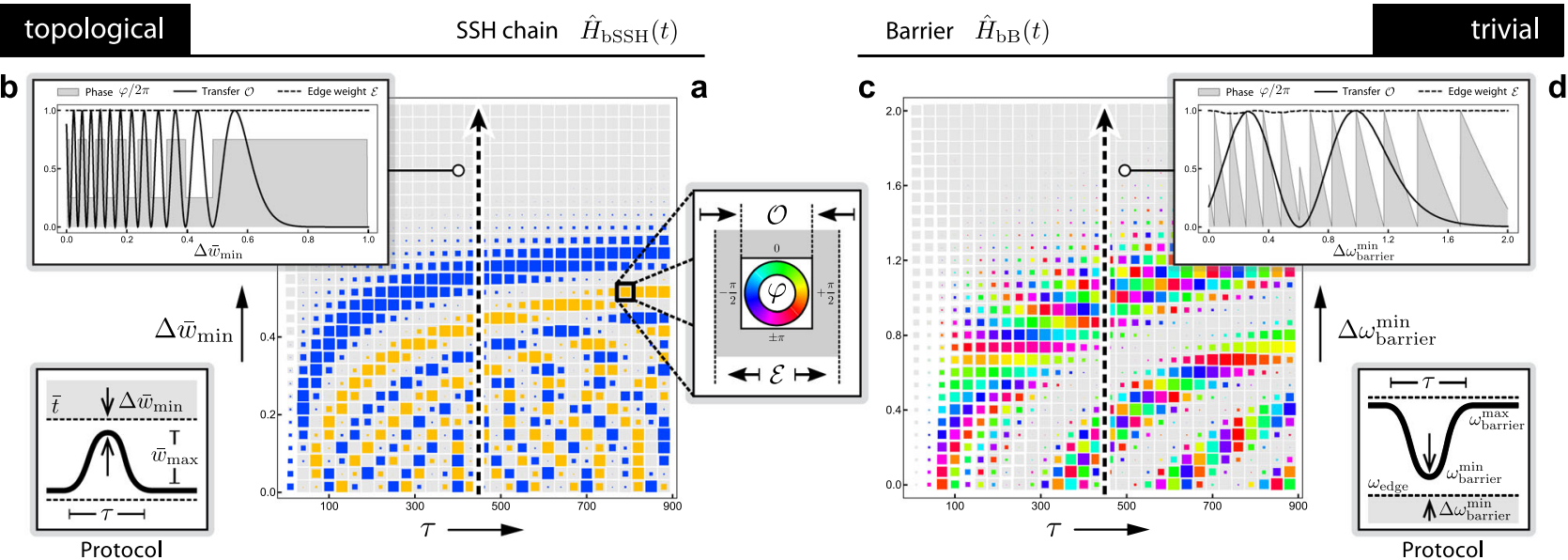

Fig. 4 State transfer-quantitative results. In a, c we plot the figures of merit for transfers driven by $\hat{H}_{\mathrm{bSSH}}(t)$ and $\hat{H}_{\mathrm{bB}}(t)$ in dependence of the protocol timescale $\tau$ and the distance from criticality, namely $\Delta \bar{w}_{\min }=\bar{t}-\bar{w}_{\max }$ and $\Delta \omega_{\text {barrier }}^{\min }=\omega_{\mathrm{barrier}}^{\min }-\omega_{\text {edge. The diameter of the gray }}$ background squares encodes the edge weight $\mathcal{E}$; a thinning of the gray background tiling therefore indicates a loss of adiabaticity. In the shown parameter regimes, however, the edge weight is almost everywhere close to unity as there is barely any loss to bulk excitations (except for regions of fast protocols close to criticality). The diameter (color) of the colored squares encodes the transfer $\mathcal{O}$ (phase $\varphi$ ) after the protocol reached its final state ( $\varphi$ is measured in the rotating frame of the localized edge modes). a shows results for a topological SSH setup of size $L=$ 5. c shows the corresponding data for a trivial tunneling barrier setup of size $L=5$. In $\mathbf{b}$, $\mathbf{d}$ we plot $\mathcal{O}, \varphi$, and $\mathcal{E}$ along the dashed slices in $\mathbf{a}$, $\mathbf{c}$, respectively. Note that the phase is fixed for the topological setup: $\varphi= \pm \pi / 2$

with $\Delta \bar{W}^{\prime} / L=\bar{t}-\bar{W}$ measuring the distance to the topological phase transition. The lowest two solutions $\lambda_{0}^{\prime}$ and $\lambda_{1}^{\prime}$ of Eq. (13) determine the relevant energies $\Delta E_{\text {edge }}=2 \lambda_{0}^{\prime} / L$ and $\Delta E_{\text {bulk }}=\left|\lambda_{1}^{\prime}-\lambda_{0}^{\prime}\right| / L$, see Fig. $5 a$, b. Notably, both energies scale as $\sim 1 / L$ with the result that their ratio $R=\Delta E_{\text {bulk }} / \Delta E_{\text {edge }}$ saturates for large $L$. For a fixed ratio $R$ of the two energy scales, it is therefore required to approach the critical point as $\bar{t}-\bar{W} \sim 1 / L$. E. g., for $R=10$ one finds $\bar{t}-\bar{W} \approx 3.3 / L$, see Fig. $5 \mathrm{~b}$.

This result demonstrates that if one requires an adiabatic protocol $\bar{W}(t)$ with a fixed minimum ratio $R^{\min }=\Delta E_{\text {bulk }}^{\min } / \Delta E_{\text {edge }}^{\max }$ at the minimal distance $\Delta \bar{W}_{\min }=\bar{t}-\bar{W}_{\max }$ from the critical coupling, then the time $\tau$ for the protocol scales as $\tau \sim L$; see Fig. $5 c$, $d$ for simulations. The latter corresponds to the optimal scaling achievable since the Lieb-Robinson bound predicts a finite propagation speed for information. ${ }^{56}$

However, we still need to adiabatically decouple bulk from edge modes, since losses to the bulk cannot be refocused in edge modes via a global tuning of parameters. A common (and conservative) estimate for adiabaticity then reads $\tau \gtrsim\left(\Delta E_{\text {bulk }}^{\min }\right)^{-2}$, which leads to the non-optimal scaling condition $\tau \sim L^{2}$. We demonstrate in the following, that a much better scaling is achievable by a rigorous estimation of the adiabaticity condition. To this end, we parametrize the time with $s=t / \tau, s \in[0,1]$ and make the ansatz $\bar{w}(t)=\bar{w}_{\text {max }} \cdot \mathcal{P}(s)$, where the generic pulse $\mathcal{P}$ : $[0,1] \rightarrow[0,1]$ and its first derivative vanish for $s=0,1$, and $\mathcal{P}(1 / 2)=1$.

Then, the non-adiabatic losses to the bulk can be rigorously upper bounded ${ }^{57}$ by

$1-\mathcal{E} \leq\left(\frac{C_{L}[\mathcal{P}]}{\tau}\right)^{2}$

where

$C_{L}[\mathcal{P}]=\int_{0}^{1} \mathrm{~d} s \frac{C_{1}\left|\mathcal{I}^{\prime \prime}\right|}{\left(\varepsilon_{L}+\mathcal{I}\right)^{2}}+\int_{0}^{1} \mathrm{~d} s \frac{C_{2}\left|\mathcal{I}^{\prime}\right|^{2}}{\left(\varepsilon_{L}+\mathcal{I}\right)^{3}}$

with $\quad \mathcal{I} \equiv 1-\mathcal{P}, \quad C_{1,2} \quad$ numerical constants, and $\varepsilon_{L}=\Delta \bar{w}_{\min }^{\prime} /\left(L-\Delta \bar{w}_{\text {min }}^{\prime}\right)$; see the Supplementary Information Section 6 for details. Note that $\mathcal{I}(1 / 2)=0$ and $\varepsilon_{L} \sim 1 / L$ so that $C_{L}[\mathcal{P}]$ diverges for $L \rightarrow \infty$ in general. In order to bound the bulk losses, the scaling of $\tau$ has to match the scaling of $C_{L}[\mathcal{P}]$.
For $\mathcal{P}(s)=\mathcal{F}(s)=\sin ^{2}(\pi s)$ we find $C_{L}[\mathcal{F}] \sim L^{1+\frac{1}{2}}$ so that a scaling of $\tau \sim L^{1+\frac{1}{2}}$ is necessary for bulk-edge decoupling in the limit of long chains, see Fig. 5e. This is better than the quadratic scaling expected from the minimal gap $\Delta E_{\text {bulk }}^{\min }$. Unfortunately, the optimal scaling $\tau \sim L$ allowed by the Lieb-Robinson bound cannot be reached by the unoptimized pulse $\mathcal{F}$. However, in Supplementary Information Section 6 we prove that there is a sequence of polynomial pulses $\mathcal{P}_{n}$ such that $C_{L}\left[\mathcal{P}_{n}\right] \sim L^{1+\frac{1}{n}}$ for $n \geq 2$ even integers, i.e., the scaling can be drastically improved by pulse optimization so that linear scaling can be approached to an arbitrary degree. Numerical simulations of the bulk losses for various scalings of $\tau$ reveal that they indeed follow the prescribed scaling of the rigorous upper bounds, see Fig. $5 f$. As a final remark, we stress that the coefficients in $C_{L}\left[\mathcal{P}_{n}\right]$ become larger with $n$, i.e., there is a pay-off between scaling and offset. Thus one may even benefit from pulses with poor scaling if only chains of fixed length are considered.

\section{Benchmarking against topologically trivial setups}

To unveil the characteristic features of the topological setup, we contrast it with two similar but topologically trivial networks, see Fig. 2c, d. The simplest approach to envisage is based on initially decoupled modes at fixed frequency $\omega_{l} \equiv \bar{\omega}$, a homogeneous tuning of all couplings $w_{i}=t_{i} \equiv \bar{t}(t)=\bar{t}_{\text {max }} \mathcal{F}(t)$, and employs the free bulk propagation of the initially localized edge modes. As shown in Fig. 3d, this approach fails to relocalize the excitation at the opposite edge due to the propagation via bulk modes; such a protocol would require either fine tuning of the pulse shape via optimal control and/or local addressability of all couplings within the network. It is therefore not competitive against the topological setup.

A more sophisticated approach mimics the presence of localized edge modes by a large tunnel barrier: The two modes at the edge have fixed frequency $\omega_{1}=\omega_{\bar{L}}=\omega_{\text {edge }}=$ const, and are separated from each other by a "potential barrier" of modes with tunable frequencies $\omega_{\text {barrier }}(t)$ and fixed couplings $\bar{t}=\bar{w}$. In analogy to the topological setup, this network exhibits exponentially localized edge modes. Transfer is again achieved by lowering the excitation gap to the bulk modes to allow for tunneling 
a

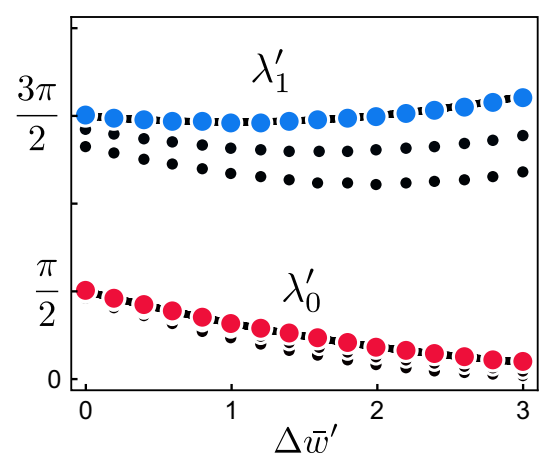

C

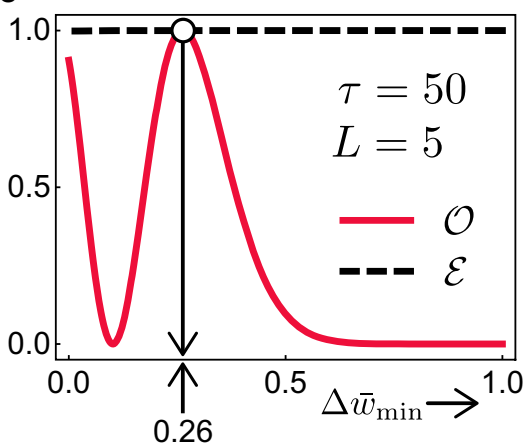

e

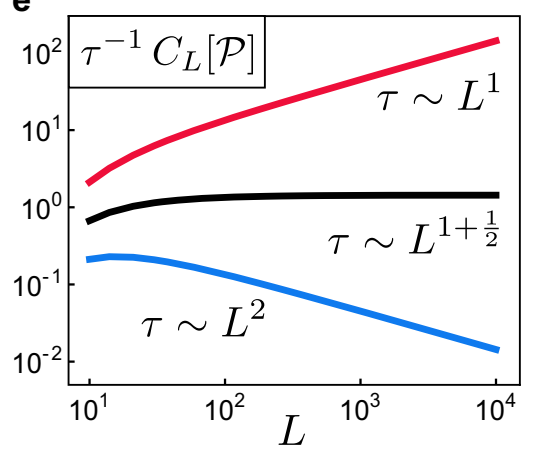

b

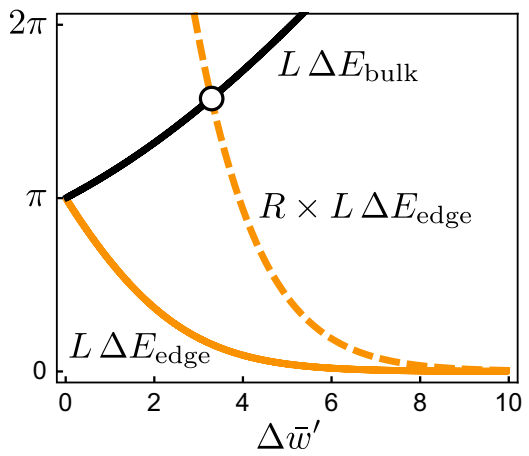

d

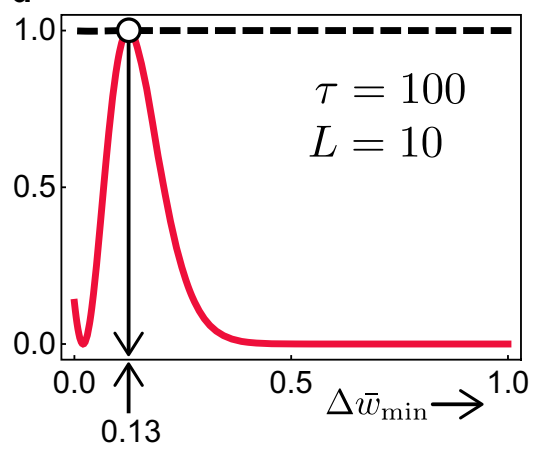

f

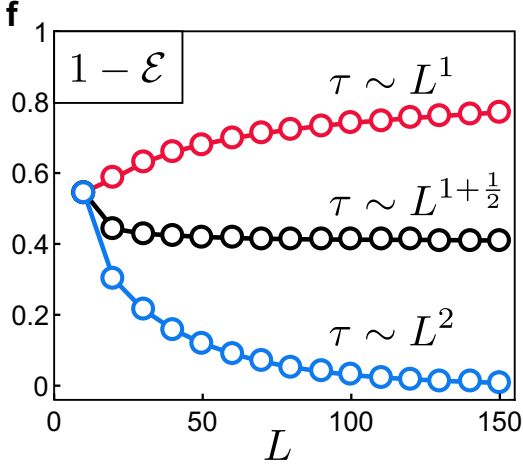

Fig. 5 Scaling and adiabaticity. a Rescaled lowest eigenvalues $\lambda_{i}^{\prime}(i=0,1)$ of $H_{\mathrm{bSSH}}$ as function of the rescaled coupling $\Delta \bar{w}^{\prime}=L(\bar{t}-\bar{w})$. Solid lines denote solutions of Eq. (13) exact for $L \rightarrow \infty$, whereas circles denote finite size results for $L=5,10$ (black) and $L=200$ (red/blue). b Energy scales $L \Delta E_{\text {edge }}=2 \lambda_{0}^{\prime}$ (solid yellow) and $L \Delta E_{\text {bulk }}=\left|\lambda_{1}^{\prime}-\lambda_{0}^{\prime}\right|$ (solid black) for $L \rightarrow \infty$ calculated from the results in a. Fixing the ratio $R=\Delta E_{\text {bulk }} /$ $\Delta E_{\text {edge }}$ (here $R=10$ ) determines $\Delta \bar{W}^{\prime}$ via the intersection marked with a circle (here $\Delta \bar{W}^{\prime} \approx 3.3$ ). c Simulations of transfer fidelity $\mathcal{O}$ (solid red) and edge weight $\mathcal{E}$ (dashed black) for system size $L=5$, protocol timescale $\tau=50$ and pulse $\bar{w}(t)=\left(\bar{t}-\Delta \bar{W}_{\text {min }}\right) \cdot \mathcal{F}(t)$ as function of $\Delta \bar{W}_{\text {min }}$. Optimal transfer for fixed $L$ and $\tau$ is found numerically for $\Delta \bar{w}_{\min } \approx 0.26$ with bulk loss $1-\mathcal{E} \approx 1 \cdot 10^{-4}$. d The same as in c for doubled size $L=$ 10 and timescale $\tau=100$. Now, optimal transfer is achieved for $\Delta \bar{W}_{\min } \approx 0.26 / 2=0.13$ with bulk loss $1-\mathcal{E} \approx 2 \cdot 10^{-5}$. e Rigorous upper bounds $\tau^{-1} C_{L}[\mathcal{P}]$ for $\mathcal{P}(s)=\mathcal{F}(s)=\sin ^{2}(\pi s)$ and $\tau=\tau_{0} \cdot L^{1+\alpha}$ with $a=0, \frac{1}{2}, 1$ and $\tau_{0}=100, \Delta \bar{W}_{\min }^{\prime}=3.3$. A scaling $\tau \sim L^{1+\frac{1}{2}}$ yields constant bulk losses for $L \rightarrow \infty$. f Simulations of the bulk losses $0 \leq 1-\mathcal{E} \leq 1$ for the parameters in e without tuning for optimal transfer. We find that the scaling follows the corresponding upper bounds. Note that the loss was chosen large $(\sim 50 \%$ for $L=10)$ for illustrative purposes and can be controlled via $\tau_{0}$ (here $\tau_{0}=1,0.3,0.1$ for $a=0, \frac{1}{2}, 1$ )

between the edges. The protocol of this scheme reads

$\omega_{\text {barrier }}(t)=\omega_{\text {barrier }}^{\max }+\left(\omega_{\text {barrier }}^{\min }-\omega_{\text {barrier }}^{\max }\right) \cdot \mathcal{F}(t)$

where $\omega_{\text {barrier }}^{\max } \gg \omega_{\text {edge }}$ will be kept fixed and $\omega_{\text {barrier }}^{\min }>\omega_{\text {edge }}$ is a tunable protocol parameter. The bosonic network Hamiltonian of this scheme is denoted by $\hat{H}_{\mathrm{bB}}(t)$.

As shown in Fig. 3c, the tunneling approach still allows for near perfect transfer for optimal parameters and long times. However, the quantitative comparison in Fig. $4 \mathrm{c}$ shows that the trivial tunneling approach requires longer time scales of the protocol and is more sensitive to bulk losses. Even then the adiabatic decoupling is much harder to achieve with $\hat{H}_{\mathrm{bB}}(t)$ than with $\hat{H}_{\text {bSSH }}(t)$, as the plots of $\mathcal{E}$ along the dashed cuts in Fig. $4 \mathrm{~b}, \mathrm{~d}$ reveal.

However, the most striking difference is the phase accumulated during the protocol: for the trivial setup, it is highly sensitive to both parameters. This is expected for a generic adiabatic protocol and is in stark contrast to the topological setup. The reason for this qualitative difference is rooted in the $\mathrm{PH}$ symmetry of the $\mathrm{SSH}$ setup which gives rise to the symmetric band structure depicted in Fig. 3a, as opposed to the asymmetric band structure of the barrier setup in Fig. 3c. As a consequence, we find that even for the ideal, topologically trivial setup, adiabatic protocols are 
a
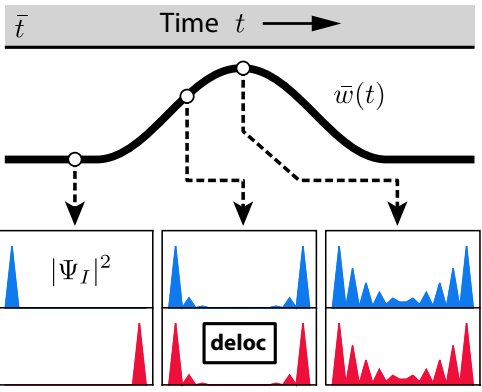

b

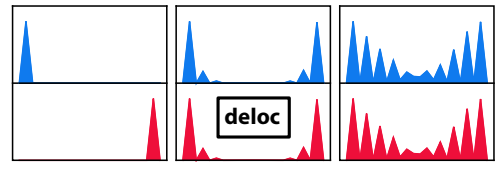

C
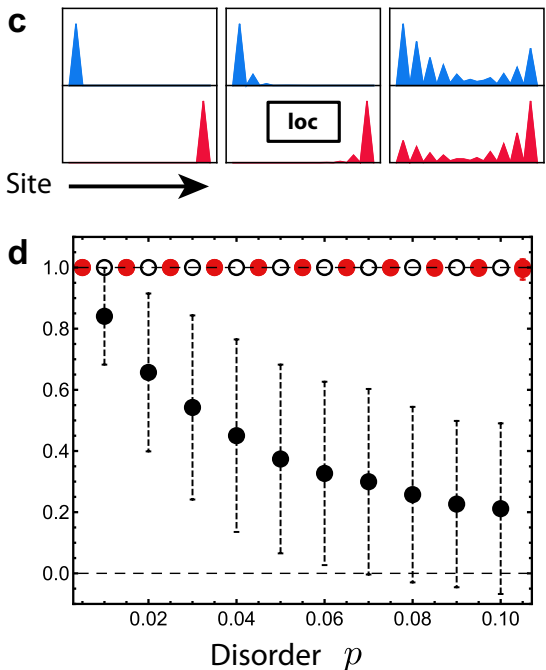

$\mathrm{PH}$ break. $\langle\langle\mathcal{O}\rangle\rangle$
$\mathrm{PH}$ sym. $\langle\langle\mathcal{O}\rangle\rangle$
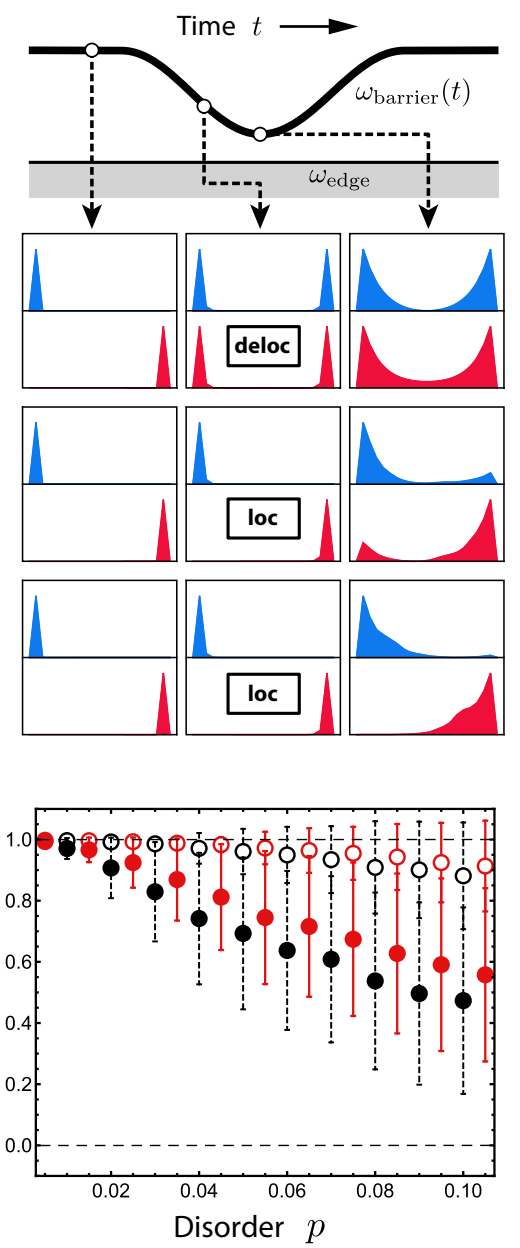

O PH break. $\langle\langle\mathcal{E}\rangle\rangle$

O PH sym. $\langle\langle\mathcal{E}\rangle\rangle$

Fig. 6 Effects of disorder. In a-c we show the spatial amplitudes of the two edge modes (blue and red) at three different times during the protocols of the SSH setup (left) and the barrier setup (right) for a no disorder, b PH symmetric disorder, and $\mathbf{c}$ PH breaking disorder. Note the (de-) localization of the edge states for PH symmetric disorder in b. In d we show the transfer $\langle\langle\mathcal{O}\rangle\rangle$ (bullets) and edge weight $\langle\langle\mathcal{E}\rangle\rangle$ (circles) for $\mathrm{PH}$ breaking (black) and symmetric (red) disorder. The averages are computed from $N=1000$ samples for a chain of length $L=5$ with a retuning of $\tau$ for every single disorder realization to optimize transfer. The error bars denote one standard deviation of the sample

unsuitable as the sensitivity of the phase increases for longer wires: a transfer preserving quantum coherence requires fine tuning of the shape of the transfer pulse. This effect becomes even more drastic in the presence of disorder.

\section{Effects of disorder and symmetry protection}

The unique features of the topological setup become even more apparent in the presence of disorder and/or imperfections in the preparation. Here, we focus on quenched disorder on the time scales for a transfer. The disorder is described as Gaussian noise with dimensionless standard deviation $p$ acting on the parameters in the Hamiltonian, i.e., for the onsite hopping we have $\left\langle\left\langle w_{i}\right\rangle\right\rangle=\bar{w}$ and $\left\langle\left\langle w_{i}^{2}-\bar{w}^{2}\right\rangle\right\rangle=p^{2} \bar{w}^{2}$ with $\langle\langle\bullet\rangle\rangle$ the disorder average. In the following, two classes of disorder will be of interest: $P H$ symmetric disorder affects only mode couplings, but assumes perfect mode frequencies; recall Eq. (8). In contrast, $P H$ breaking disorder affects both mode couplings and frequencies.

For the topologically trivial setup, both types of disorder give rise to Anderson localization of the "artificial" edge modes, see
Fig. 6a-c. The transfer protocol, however, relies on the delocalization of the edge modes, which is prohibited by Anderson localization. As a consequence, disorder leads to a significant reduction of transfer fidelity and increased bulk losses, Fig. $6 \mathrm{~d}$. Furthermore, the phase $\varphi$ accumulated during the transfer strongly fluctuates for each disorder realization; more details on this aspect are given in Supplementary Information Section 4.

In contrast, for the topological SSH setup, the $\mathrm{PH}$ symmetric disorder respects the protecting symmetry. Then, Anderson localization of the edge modes is forbidden by a topological obstruction ${ }^{11,13,14}$ and the required overlap between the two edge modes can be established, see Fig. $6 \mathrm{~b}$. As a consequence, the transfer can still be performed perfectly with a fixed phase $\varphi= \pm \pi /$ 2. However, this requires that for each disorder realization one is allowed to adapt the transfer time $\tau$ of the protocol. In an experimental setup this corresponds, for example, to imperfections in sample preparation, which can be overcome by calibrating the setup and the transfer protocol beforehand. In turn, the $P H$ breaking disorder leads also in the topological setup to localization of the edge modes and a reduction of transfer fidelity, see Fig. $6 \mathrm{~d}$. 


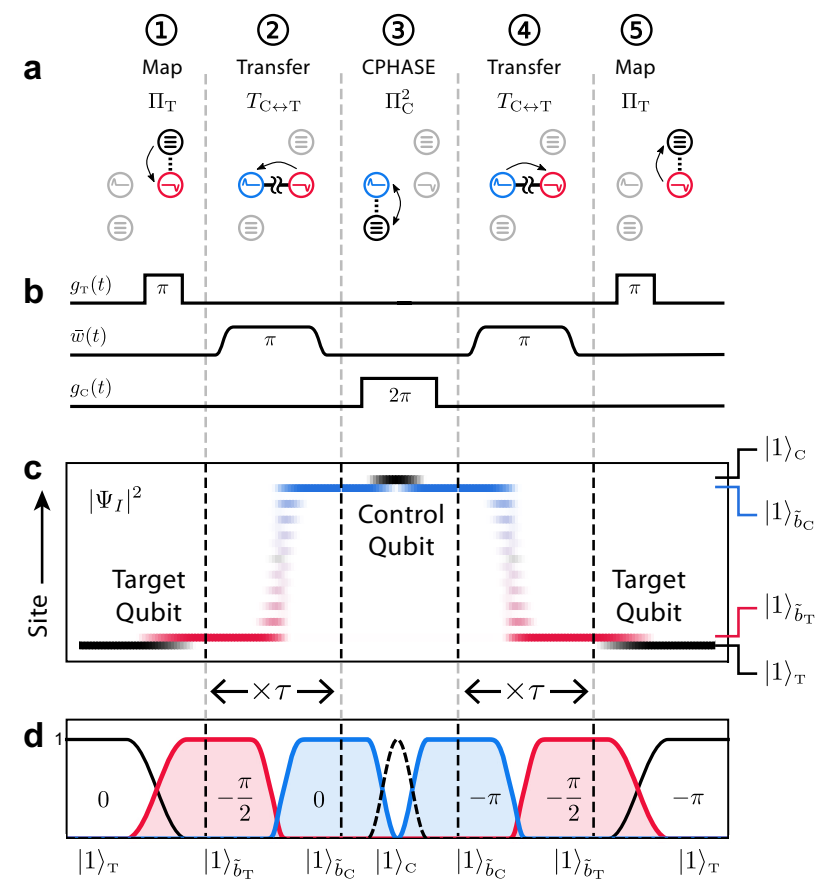

Fig. 7 CP gate-pulse sequence and results. a Schematic illustration of the five steps needed to perform the CP gate $\mathcal{U}_{\mathrm{CP}}$ (3) on two remote qubits, see setup in Fig. $1 \mathrm{a}, \mathrm{b}$. b The complete pulse sequence consisting of two $\pi$ pulses $\Pi_{\mathrm{T}}$ to map the target qubit to and from edge mode $\tilde{b}_{T}$, a $2 \pi$ pulse $\Pi_{C}^{2}$ to perform the actual CP gate, and two-edge mode tunneling pulses $T_{\mathrm{C} \leftrightarrow \mathrm{T}}$. c Numerical singleparticle evolution for the two-qubit basis state $|1\rangle_{C}|1\rangle_{T}$ for a chain of length $L=10$. The density plot encodes the squared single-particle amplitude, where the upper and lower edges correspond to the logical states $|1\rangle_{C}$ and $|1\rangle_{\mathrm{T}}$ with their adjacent boundary modes $|1\rangle_{\tilde{b}_{c}}=|1,0,0\rangle=\tilde{b}_{c}^{\dagger}|0\rangle$ and $|1\rangle_{\tilde{b}_{T}}=|0, \mathbf{0}, 1\rangle=\tilde{b}_{T}^{\dagger}|0\rangle$. d Square of the absolute value of the overlaps with $|1\rangle_{T}$ (solid black), $|1\rangle_{\tilde{b}_{T}}$ (solid red), $|1\rangle_{\tilde{b}_{C}}$ (solid blue), and $|1\rangle_{C}$ (dashed black). The relative phases w.r.t. the target qubit are shown as insets. Note that the scales for the Rabi pulses and the topological state transfer differ by a factor of $\tau$

\section{Application: CP gate}

As an application, we demonstrate how the proposed state transfer, protected by $\mathrm{PH}$ symmetry, can be employed for a $\mathrm{CP}$ gate between two remote qubits that are coupled to the local edge modes of the topological SSH network. The complete protocol for a $\mathrm{CP}$ gate between the target qubit $\mathrm{T}$ and the control qubit $C$ is based on a well-known scheme that makes use of auxiliary levels $|a\rangle_{\mathrm{T} / \mathrm{C}}{ }^{58}{ }^{5}$ We focus on the setup shown in Fig. $1 \mathrm{~b}$ for the qubits and the auxiliary states with the coupling Hamiltonian between the qubits and the edge modes of the $\mathrm{SSH}$ chain given by Eq. (2). The full protocol for the CP gate follows the procedure in Eq. (3) and is described in the following, see Fig. 7.

First, the full sequence $\mathcal{U}_{C P}$ leaves the states $|0\rangle_{C}|0\rangle_{T}$ and $|1\rangle_{C}|0\rangle_{T}$ invariant because there are no excitations in the network. On the other hand, for $|0\rangle_{C}|1\rangle_{T}$ and $|1\rangle_{C}|1\rangle_{T}$ the first $\pi$-pulse $\Pi_{T}$ on the target qubit maps the state $|1\rangle_{\mathrm{T}}$ to a bosonic excitation in the right edge mode $\tilde{b}_{T}$ with the phase $-\pi / 2$. It is important that this operation is performed slowly compared to the energy gap to bulk excitations in the SSH chain: Then, energy conservation allows one to only address the coupling to the edge states and suppress admixture of bulk excitations. The subsequent transfer of the excitation to the left edge $T_{\mathrm{\leftrightarrow} \leftrightarrow \mathrm{T}}$ implies an additional phase $\pi /$ $2+L \pi$. The full Rabi cycle $\Pi_{C}^{2}$ provides a phase $\pi$ if and only if the control qubit is in state $|1\rangle_{\mathrm{C}}$. The subsequent transfer back $T_{\mathrm{C} \leftrightarrow \mathrm{T}}$ and the $\pi$-pulse $\Pi_{\mathrm{T}}$ provide additional phases $\pi / 2+L \pi$ and $-\pi / 2$. Therefore, the full protocol implements the mapping $|1\rangle_{C}|1\rangle_{T} \rightarrow$
$-|1\rangle_{C}|1\rangle_{T}$ while all orthogonal states remain invariant. Given quantum coherence during the protocol, this realizes a controlled phase gate with phase $\pi$. A full numerical time evolution for the state $|1\rangle_{C}|1\rangle_{T}$ is shown in Fig. 7c, d, confirming the above argumentation.

Finally, we point out that the linearity of the network implies that the transfer takes place for each excitation of edge modes independently. I.e., if $\left|n_{\mathrm{C}}, n_{T}\right\rangle$ denotes the state with $n_{\mathrm{C}}$ excitations in the left edge mode $\tilde{b}_{C}$ and $n_{T}$ excitations in the right edge mode $\tilde{b}_{\mathrm{T}}$, the transfer operation $T_{\mathrm{C} \leftrightarrow \mathrm{T}}$ implements the mapping $\left|n_{\mathrm{C}}, n_{\mathrm{T}}\right\rangle \rightarrow( \pm i)^{n_{\mathrm{C}}+n_{\mathrm{T}}}\left|n_{\mathrm{T}}, n_{\mathrm{C}}\right\rangle$ (for even/odd L). This observation immediately implies that the unitary operation $\mathcal{U}_{\text {SWAP }}$ in Eq. (4) swaps the qubits.

\section{Extension to 2D networks of coupled qubits}

An important aspect of the SSH chain is its symmetry class BDI (due to the real hopping amplitudes $w_{i}$ and $t_{i}$ ), so that the setup is characterized by a $\mathbb{Z}$ topological invariant. This allows us to extend the analysis to two-dimensional setups by placing several $\mathrm{SSH}$ chains parallel to each other and adding couplings between them, see Fig. 1c for a possible realization. As long as these couplings are real and respect the sublattice symmetry, the setup is still topologically protected and each chain endpoint carries an edge mode. Possible relaxations of the symmetry constraints are presented in Supplementary Information Section 3.

Due to the sublattice symmetry, there are two types of modes, "even" and "odd" ones, depending on how they transform under $S=U_{C}^{S S H}$ [recall Eq. (6)]. Edge mode pairs of different types can communicate efficiently by tuning the couplings along a connecting path that resembles the one-dimensional SSH setup; this is illustrated in Fig. 1c. Because of the bulk gap, this procedure is very robust and couplings that deviate from the desired path ("stray couplings") have no detrimental effect on the state transfer, as long as they respect the symmetries and do not couple to other edge modes. This can be guaranteed by a modification of the setup such that qubits and edge modes are relocated at the end of one-dimensional chains that emanate from the 2D network, see Fig. 1d. Remarkably, the setup allows for an enhancement of the edge mode overlap by tuning the couplings of the 2D bulk globally instead of tracing out a particular path that connects the qubits. Therefore, the minimal experimental requirement is the individual addressability of each branch that connects an edge mode to the 2D bulk. Since the edge modes can now be separated, this constraint is very weak, and in general already satisfied by the requirement of local gate operations on the qubit.

Note that coupling edge modes of the same type is obstructed by the sublattice symmetry, as indicated by the lower path in Fig. 1c and proven in Supplementary Information Section 3. However, the implementation of an exchange of qubits via $\mathcal{U}_{\text {SWAP }}$ facilitates the application of the controlled phase gate $\mathcal{U}_{\mathrm{CP}}$ between any pair in the network: If the two qubits couple to edge modes of different types, one can directly perform the $\mathrm{CP}$ gate between them. Conversely, if the qubits couple to edge modes of the same type, one first performs an exchange $\mathcal{U}_{\text {SWAP }}$ with an arbitrary qubit of the opposite type, applies the CP gate, and maps the qubit back by another exchange.

\section{DISCUSSION}

We have demonstrated that a topological network consisting of linearly coupled bosonic degrees of freedom, capable of carrying single, quantized excitations, allows for efficient quantum communication between distant qubits. Weak addressability of each branch within the network is sufficient and no local addressability of individual sites is required. Remarkably, the time scale for the operations scales almost linearly with the distance between the qubits. Furthermore, topological protection guarantees robustness 
against quenched disorder in the setup by evading Anderson localization of the edge modes. In summary, we have shown that the unique properties of (quasi) one-dimensional topological systems can be harvested for efficient quantum communication between qubits. These benefits come with the price of higher complexity in realization and preparation as the coupling parameters have to respect the symmetries protecting the topological invariants.

\section{METHODS}

For more details on the derivations, see the Supplementary Information.

Data availability

The numerical code and the datasets used for this study are available from the corresponding author on reasonable request.

\section{ACKNOWLEDGEMENTS}

This research has received funding from the European Research Council (ERC) under the European Union's Horizon 2020 research and innovation program (grant agreement No. 681208). This research was supported in part by the National Science Foundation under Grant No. NSF PHY-1125915.

\section{AUTHOR CONTRIBUTIONS}

All authors contributed to the paper equally.

\section{ADDITIONAL INFORMATION}

Supplementary information accompanies the paper on the npj Quantum Information website (https://doi.org/10.1038/s41534-017-0047-x).

Competing interests: The authors declare no competing financial interests.

Publisher's note: Springer Nature remains neutral with regard to jurisdictional claims in published maps and institutional affiliations.

\section{REFERENCES}

1. Nayak, C., Simon, S. H., Stern, A., Freedman, M. \& Sarma, S. D. Non-abelian anyons and topological quantum computation. Rev. Mod. Phys. 80, 1083-1159 (2008).

2. Ando, T., Matsumoto, Y. \& Uemura, Y. Theory of hall effect in a two-dimensional electron system. J. Phys. Soc. Jpn. 39, 279-288 (1975).

3. v. Klitzing, K., Dorda, G. \& Pepper, M. New method for high-accuracy determination of the fine-structure constant based on quantized hall resistance. Phys. Rev. Lett. 45, 494-497 (1980).

4. Laughlin, R. B. Quantized hall conductivity in two dimensions. Phys. Rev. B 23 5632-5633 (1981).

5. Tsui, D. C., Stormer, H. L. \& Gossard, A. C. Two-dimensional magnetotransport in the extreme quantum limit. Phys. Rev. Lett. 48, 1559-1562 (1982).

6. Stormer, H. L. et al. Fractional quantization of the hall effect. Phys. Rev. Lett. $\mathbf{5 0}$ 1953-1956 (1983).

7. Willett, R. et al. Observation of an even-denominator quantum number in the fractional quantum hall effect. Phys. Rev. Lett. 59, 1776-1779 (1987).

8. Konig, M. et al. Quantum spin hall insulator state in HgTe quantum wells. Science 318, 766-770 (2007).

9. Hsieh, D. et al. A topological dirac insulator in a quantum spin hall phase. Nature 452, 970-974 (2008).

10. $\mathrm{Xu}, \mathrm{Y}$. et al. Observation of topological surface state quantum hall effect in an intrinsic three-dimensional topological insulator. Nat. Phys. 10, 956-963 (2014).

11. Schnyder, A. P., Ryu, S., Furusaki, A. \& Ludwig, A. W. W. Classification of topological insulators and superconductors in three spatial dimensions. Phys. Rev. B 78, 195125 (2008).

12. Kitaev, A. Periodic table for topological insulators and superconductors. In AIP Conference Proceedings, Vol. 1134, 22-30 (AIP, 2009).

13. Schnyder, A. P., Ryu, S., Furusaki, A. \& Ludwig, A. W. W. Classification of topological insulators and superconductors. In AIP Conference Proceedings, Vol. 1134 10-21 (2009).
14. Ryu, S., Schnyder, A. P., Furusaki, A. \& Ludwig, A. W. W. Topological insulators and superconductors: tenfold way and dimensional hierarchy. New J. Phys. 12, 065010 (2010)

15. Hasan, M. Z. \& Kane, C. L. Colloquium: topological insulators. Rev. Mod. Phys. 82, 3045-3067 (2010).

16. Qi, X.-L. \& Zhang, S.-C. Topological insulators and superconductors. Rev. Mod. Phys. 83, 1057-1110 (2011).

17. Wigner, E. P. On the statistical distribution of the widths and spacings of nuclear resonance levels. Math. Proc. Camb. Philos. Soc. 47, 790 (1951).

18. Wigner, E. P. On the distribution of the roots of certain symmetric matrices. Ann. Math. 67, 325 (1958)

19. Dyson, F. J. The threefold way. algebraic structure of symmetry groups and ensembles in quantum mechanics. J. Math. Phys. 3, 1199-1215 (1962).

20. Altland, A. \& Zirnbauer, M. R. Nonstandard symmetry classes in mesoscopic normal-superconducting hybrid structures. Phys. Rev. B 55, 1142-1161 (1997).

21. Hafezi, M., Mittal, S., Fan, J., Migdall, A. \& Taylor, J. M. Imaging topological edge states in silicon photonics. Nat. Photon. 7, 1001-1005 (2013).

22. Susstrunk, R. \& Huber, S. D. Observation of phononic helical edge states in a mechanical topological insulator. Science 349, 47-50 (2015).

23. Nash, L. M. et al. Topological mechanics of gyroscopic metamaterials. Proc. Natl. Acad. Sci. 112, 14495-14500 (2015).

24. Rechtsman, M. C. et al. Photonic floquet topological insulators. Nature 496 196-200 (2013).

25. Ling, C. W., Xiao, M., Chan, C. T., Yu, S. F. \& Fung, K. H. Topological edge plasmon modes between diatomic chains of plasmonic nanoparticles. Opt. Express 23 2021 (2015).

26. Ningyuan, J., Owens, C., Sommer, A., Schuster, D. \& Simon, J. Time- and siteresolved dynamics in a topological circuit. Phys. Rev. X 5, 021031 (2015).

27. Süsstrunk, R., Zimmermann, P. \& Huber, S. D. Switchable topological phonon channels. New J. Phys. 19, 015013 (2017).

28. Atala, M. et al. Direct measurement of the zak phase in topological bloch bands. Nat. Phys. 9, 795-800 (2013).

29. Jotzu, G. et al. Experimental realization of the topological haldane model with ultracold fermions. Nature 515, 237-240 (2014).

30. Aidelsburger, $M$. et al. Measuring the chern number of hofstadter bands with ultracold bosonic atoms. Nat. Phys. 11, 162-166 (2014).

31. Mancini, M. et al. Observation of chiral edge states with neutral fermions in synthetic hall ribbons. Science 349, 1510-1513 (2015).

32. Stuhl, B. K., Lu, H.-I., Aycock, L. M., Genkina, D. \& Spielman, I. B. Visualizing edge states with an atomic bose gas in the quantum hall regime. Science 349 1514-1518 (2015).

33. Duca, L. et al. An aharonov-bohm interferometer for determining bloch band topology. Science 347, 288-292 (2015).

34. Lohse, M., Schweizer, C., Zilberberg, O., Aidelsburger, M. \& Bloch, I. A thouless quantum pump with ultracold bosonic atoms in an optical superlattice. Nat. Phys. 12, 350-354 (2015).

35. Haldane, F. D. M. \& Raghu, S. Possible realization of directional optical waveguides in photonic crystals with broken time-reversal symmetry. Phys. Rev. Lett. 100, 013904 (2008)

36. Koch, J., Houck, A. A., Hur, K. L. \& Girvin, S. M. Time-reversal-symmetry breaking in circuit-QED-based photon lattices. Phys. Rev. A 82, 043811 (2010).

37. Hafezi, M., Demler, E. A., Lukin, M. D. \& Taylor, J. M. Robust optical delay lines with topological protection. Nat. Phys. 7, 907-912 (2011).

38. Berg, N., Joel, K., Koolyk, M. \& Prodan, E. Topological phonon modes in filamentary structures. Phys. Rev. E 83, 021913 (2011).

39. Yannopapas, V. Topological photonic bands in two-dimensional networks of metamaterial elements. New J. Phys. 14, 113017 (2012)

40. Kane, C. L. \& Lubensky, T. C. Topological boundary modes in isostatic lattices. Nat. Phys. 10, 39-45 (2013).

41. Lu, L., Joannopoulos, J. D. \& Soljačić, M. Topological photonics. Nat. Photon. 8, 821-829 (2014).

42. Kariyado, T. \& Hatsugai, Y. Manipulation of dirac cones in mechanical graphene. Sci. Rep. 5, 18107 (2015).

43. Wang, Y.-T., Luan, P.-G. \& Zhang, S. Coriolis force induced topological order for classical mechanical vibrations. New J. Phys. 17, 073031 (2015).

44. Wang, P., Lu, L. \& Bertoldi, K. Topological phononic crystals with one-way elastic edge waves. Phys. Rev. Lett. 115, 104302 (2015).

45. Peter, D. et al. Topological bands with a chern number $\mathrm{C}=2$ by dipolar exchange interactions. Phys. Rev. A 91, 053617 (2015).

46. Yao, $\mathrm{N}$. et al. Topologically protected quantum state transfer in a chiral spin liquid. Nat. Commun. 4, 1585 (2013).

47. Dlaska, C., Vermersch, B. \& Zoller, P. Robust quantum state transfer via topologically protected edge channels in dipolar arrays. Quantum Sci. Technol. 2, 015001 (2017). 
48. Zak, J. Berry's phase for energy bands in solids. Phys. Rev. Lett. 62, 2747-2750 (1989).

49. Kitaev, A. Y. Unpaired majorana fermions in quantum wires. Phys.-Usp. 44, 131-136 (2001).

50. Su, W. P., Schrieffer, J. R. \& Heeger, A. J. Solitons in polyacetylene. Phys. Rev. Lett. 42, 1698-1701 (1979).

51. Houck, A. A., Türeci, H. E. \& Koch, J. On-chip quantum simulation with superconducting circuits. Nat. Phys. 8, 292-299 (2012).

52. Carusotto, I. \& Ciuti, C. Quantum fluids of light. Rev. Mod. Phys. 85, 299-366 (2013).

53. Lukin, M. D. et al. Dipole blockade and quantum information processing in mesoscopic atomic ensembles. Phys. Rev. Lett. 87, 037901 (2001).

54. Doherty, M. W. et al. The nitrogen-vacancy colour centre in diamond. Phys. Rep. 528, 1-45 (2013).

55. Duan, L.-M. \& Monroe, C. Colloquium: quantum networks with trapped ions. Rev. Mod. Phys. 82, 1209-1224 (2010).

56. Lieb, E. H. \& Robinson, D. W. The finite group velocity of quantum spin systems. Commun. Math. Phys. 28, 251-257 (1972).

57. Jansen, S., Ruskai, M.-B. \& Seiler, R. Bounds for the adiabatic approximation with applications to quantum computation. J. Math. Phys. 48, 102111 (2007).
58. Haack, G., Helmer, F., Mariantoni, M., Marquardt, F. \& Solano, E. Resonant quantum gates in circuit quantum electrodynamics. Phys. Rev. B 82, 024514 (2010).

(7) Open Access This article is licensed under a Creative Commons Attribution 4.0 International License, which permits use, sharing, adaptation, distribution and reproduction in any medium or format, as long as you give appropriate credit to the original author(s) and the source, provide a link to the Creative Commons license, and indicate if changes were made. The images or other third party material in this article are included in the article's Creative Commons license, unless indicated otherwise in a credit line to the material. If material is not included in the article's Creative Commons license and your intended use is not permitted by statutory regulation or exceeds the permitted use, you will need to obtain permission directly from the copyright holder. To view a copy of this license, visit http://creativecommons. org/licenses/by/4.0/.

(c) The Author(s) 2017 